\title{
Comparison of nutrient intake in adolescents and adults with and without food allergies
}

Kate Maslin ${ }^{1,2}$, Carina Venter ${ }^{1,3,4}$, Heather MacKenzie ${ }^{1,5}$, Berber Vlieg-Boerstra ${ }^{6}$, Tara Dean $^{1,3}$, Isolde Sommer ${ }^{1,7}$,

${ }^{1}$ University of Portsmouth, School of Health Sciences and Social Work, United Kingdom

${ }^{2}$ University of Southampton, MRC Lifecourse Epidemiology Unit, Southampton, United Kingdom.

${ }^{3}$ David Hide Asthma and Allergy Research Centre, Isle of Wight, United Kingdom

${ }^{4}$ Division of Allergy and Immunology, Cincinnati Children's Hospital Medical Centre, Cincinnati

${ }^{5}$ University of Portsmouth, Graduate School, United Kingdom

${ }^{6}$ Onze Lieve Vrouwe Gasthuis, Amsterdam, The Netherlands

${ }^{7}$ Danube University Krems, Department for Evidence-Based Medicine and Clinical Epidemiology, Austria

$\mathrm{KM}$ and IS drafted the manuscript. IS, HM, TD, and CV were involved in conception and design of the study. IS was responsible for data collection and analysis. KM, CV, HM, BV, TD and IS contributed substantially to interpretation of data. All authors critically revised the manuscript and approved its final version.

Corresponding author and requests for offprints: Isolde Sommer, MPH, PhD, Research Associate, Danube University Krems, Department of Evidence-based Medicine and Clinical Epidemiology, Dr.-Karl-Dorrek-Straße 30, 3500 Krems, Austria, T: +43 (0)2732 893 2925, F: +43 (0)2732 893-4910, Email: isolde.sommer@donau-uni.ac.at;

Short title: Nutrient intake in food allergy

Key words: food allergies, nutrient intake, supplements, children, adolescents, adults 


\begin{abstract}
Background: Exclusion diets for the management of food allergy pose a risk of nutritional deficiencies and inadequate growth in children, yet less is known about their effect in adolescents and adults. The aim of this study was to compare the dietary intake of adolescents and adults with food allergies to a control group.
\end{abstract}

Methodology: A food allergic and a control group were recruited from Portsmouth and the Isle of Wight in the United Kingdom. Participants were recruited from a food allergy charity, allergy clinics, a local school and university, and previous research studies. Macro and micronutrient intake data were obtained using a 4-day estimated food diary. Sociodemographic and anthropometric data was collected via a constructed questionnaire.

Results: This cross-sectional study included 81 adolescents (48 food allergic and 33 controls) aged 11-18y and 70 adults aged 19-65y (23 food allergic and 47 controls). Overall 19 (22.8\%) adolescents and $19(27.1 \%)$ adults took dietary supplements, with no difference according to food allergic status. Adolescents with food allergy had higher intakes of niacin and selenium than adolescents without $(\mathrm{p}<0.05)$. This difference persisted when dietary supplements were removed from the analysis. Adults with food allergies had higher intakes of folate and zinc than those without $(\mathrm{p}<0.05)$, however this was not observed when dietary supplements were removed from the analysis. Across all participants, the intake of several micronutrients was suboptimal. There was no difference in protein or calorie intake, or body mass index, according to food allergic status.

Conclusions: The dietary intake of food allergic participants was broadly similar and in some cases better than that of control participants. However suboptimal intakes of several micronutrients were observed across all participants, suggesting poor food choices. 


\section{Introduction}

Food allergy is defined as a reproducible adverse immune response to a food ${ }^{(1)}$. The prevalence of food allergy in Europe across all ages is estimated to be 0.6-6\%, dependent on method of diagnosis ${ }^{(2)}$. Eight foods: cows' milk, hens' egg, soy, peanuts, tree nuts, wheat, fish, and shellfish, account for more than $90 \%$ of childhood cases of food allergy ${ }^{(3)}$. Typically, adult food allergy is caused by a more diverse range of foods, including fruit and vegetables, and may present differently than in children. At present there is no cure available, management requires the individual to consume an exclusion diet avoiding the causative allergen. Therefore the main goals in the management of food allergy are to avoid the offending food(s), whilst providing an adequate, healthy and nutritionally balanced diet ${ }^{(4)}$.

A number of studies have investigated the diets of infants and children with milk and multiple food allergies, demonstrating differences in protein, calcium, zinc, vitamin D and iron intakes ${ }^{(5-7)}$. Additionally, research has shown that infants and children with cows' milk allergy (CMA) ${ }^{(8-10)}$ or multiple food allergies (to a combination of to milk, egg, wheat, soya, fish tree nut and/or peanut) are at increased risk for impaired growth ${ }^{(5,6,11-14)}$ although overweight and obesity have also been reported ${ }^{(12)}$. Less is known about the nutritional intake of adolescents and adults with food allergy. The few published studies of adult participants have reported contrasting results. For example, higher intakes of calcium, iron, folate and vitamin $\mathrm{E}$ have been demonstrated when data was combined from participants $>20$ years with food allergies to milk, egg, wheat, soya, beef, pork or chicken ${ }^{(15)}$. Conversely, lower intakes of calcium and phosphorous have been reported in young adults with CMA, with one study reporting that $27 \%$ were at risk of osteoporosis ${ }^{(16)}$. Although pollen food syndrome, a food allergy associated with birch pollen, is noted to affect $2 \%$ of adults in the UK and typically first occurs below the age of 20 years ${ }^{(17)}$, its nutritional impact has not been documented.

Although there is some heterogeneity in study designs and results, most published studies report that energy intakes are broadly similar between those with and without food allergies. In general research has indicated that excluded food groups are adequately substituted in terms of calories, although not always in terms of micronutrients ${ }^{(5,6,11,14)}$. As there is some evidence that food allergy may be increasing ${ }^{(2)}$ and the vast majority of research evidence relates to young children, there is a clear need for studies evaluating dietary intake in adolescents and adults with food allergies. Simply extrapolating findings from paediatric to older populations may lead to erroneous assumptions ${ }^{(20)}$. This is particularly 
important for those who may be consuming restricted diets during adolescence, a time of emerging independence in health behaviours, that occurs alongside a period of heightened nutritional requirements and rapid growth ${ }^{(18,19)}$. This study therefore aims to investigate dietary intake in food allergic adolescents and adults, using healthy control groups as comparison.

\section{Methods}

\section{Study design and participants}

This cross-sectional study of adolescents (11-18 years) and adults (19-65 years) took place in Hampshire and the Isle of Wight (UK) between October 2011 and May 2012. A food allergic group and a control group were recruited. Recruitment is shown in Figure 1.

Food allergic participants were recruited through an allergy support charity, local allergy clinics and previous allergy studies ${ }^{(21,22)}$. The inclusion criteria for the food-allergic participants was the presence of a confirmed diagnosis of $\operatorname{IgE}$ or non-IgE mediated allergy to at least one of the following foods: egg, milk, peanuts, tree nuts, sesame, crustaceans, fish or wheat.

A control group of healthy participants, who consumed an unrestricted diet, were recruited through local schools, advertisements on the University of Portsmouth website, and previous studies ${ }^{(21,22)}$. Participants with other conditions with dietary implications (e.g. diabetes) were excluded.

\section{Nutrient intakes}

Participants were asked to keep a record of everything eaten and drunk over four consecutive days, including one weekend day. Life size pictures of glasses and spoons, and photographs of 15 frequently consumed foods as small, medium and large portion sizes were provided ${ }^{(23)}$. Otherwise participants were asked to record household measures, number of items or weight from labels of packaged foods. In addition, they were asked to record brand names, recipes and dietary supplements. Once returned, diaries were reviewed and all participants were phoned to prompt for missing details.

Nutrient analysis was based on the UK National Nutrition Databank that integrates the sixth edition of McCance \& Widdowson's the Composition of Foods ${ }^{(24)}$ and its accompanying 
supplements. Portion sizes were estimated using UK coding criteria on food portion sizes in adults ${ }^{(25)}$, and age-appropriate portion sizes for adolescents ${ }^{(26)}$. Data were entered into Dietplan 6 (Forestfield Software Limited, Horsham, UK) by a nutritionist (IS) and two dietitians. To eliminate interpersonal variability in coding, the nutritionist checked all data for coding and portion size errors.

Questionnaires

Questionnaires were constructed to collect information on clinical and socio-demographic characteristics of participants, based on a standardised questionnaire previously used ${ }^{(27)}$. Information on anthropometric measurements and dietary supplements was also collected. The food-allergic group received a questionnaire with questions on food allergies such as foods avoided, type of allergy (single/multiple), mode of diagnosis of food allergies (skin prick test, blood test, doctor's diagnosis, food challenge), and symptoms. Questionnaires were assessed for clarity by allergy patients, nurses and dietitians.

\section{Energy reporting}

The Goldberg equation was used to identify participants who under-reported intake ${ }^{(28)}$. Physical activity levels and data required for calculating Goldberg cut-offs were taken from Black ${ }^{(29)}$. Basal metabolic rate was predicted from standard age- and gender-specific equations derived from Schofield ${ }^{(30)}$. As only extreme degrees of under-reporting can be detected with the Goldberg equation ${ }^{(31)}$, identified under-reporters were not excluded, but adjusted for in the analyses (yes/no).

\section{Statistical analysis}

Sample size calculation was based on calcium intake from a previous study ${ }^{(32)}$, which has been identified as the key at-risk nutrient ${ }^{(5,6,8,14,33)}$. The calculation yielded a target sample size of 50 in each age group, using 0.90 power, 0.05 significance level, 294mg calcium assumed variability within the population and $264 \mathrm{mg}$ as the expected difference between the two groups. Statistical analysis was carried out with IBM SPSS Statistics for Windows (version 20, Armonk, NY: IBM Corp.). All continuous variables were tested for normality. Since most of them were not normally distributed, differences between the food-allergic and control group were compared using Mann-Whitney U or $\chi^{2}$-tests. 
Data were analysed against dietary reference values for food energy and nutrients for the UK ${ }^{(34)}$. Values were expressed as percentages of estimated average requirements (EAR; $50 \%$ of the population's requirement is met) for energy intake or reference nutrient intakes (RNI; $97.5 \%$ of the population's requirement is met) for nutrients according to age and gender, and percentage of participants below and above these requirements. The significance level was set at 0.05 for all analyses.

\section{Ethical considerations}

This study was conducted according to the guidelines laid down in the Declaration of Helsinki and ethical approval was granted by the National Health Service (NHS) Southampton \& South West Hampshire Research Ethics Committee A (11/SC/0039). Written informed consent and assent was obtained from all participants.

\section{Results}

\section{Demographic characteristics and food diary completion}

This study included 151 participants, 71 of whom had food allergies. Recruitment of participants is shown in Figure 1. Sociodemographic characteristics are shown in Table 1.

Adults in the food allergy group were significantly older than the control group $(\mathrm{p}<0.05)$. Amongst adolescents, there were fewer females in the food allergy than the control group $(\mathrm{p}<$ 0.05). There were no differences in ethnicity, education level, occupation, living location or Body Mass Index (BMI) according to food allergy status. In terms of food diary completion, there was no difference in irregular reporting (i.e. weekend/consecutive days completion) or underreporting according to food allergy status. 
In the food allergy group, $70 \%$ of adolescents and $87 \%$ of adults had allergies to multiple foods. Among adolescents, peanut and tree nut allergy were the most prevalent, affecting $80 \%$ and $62 \%$ of participants respectively, followed by fruit $(22 \%)$, egg (16\%), milk (14\%) and sesame (12\%). Similarly in adults, most allergy was reported to tree nuts $(82.6 \%)$ or peanut (78.3\%), followed by vegetables $(21.4 \%)$, fruit (17.4\%) wheat (17.4\%) and shellfish (13\%). Allergies were also reported to fish, soya, sesame, pulses, seeds, sulphites, honey and rye. Specific data on the prevalence of pollen food syndrome was not collected.

\section{Dietary supplement use}

Overall 19 (22.8\%) adolescents and 19 (27.1\%) adults took dietary supplements. Of the 38 participants who took supplements, the majority $(n=22)$ took multivitamin/mineral preparations. There was no significant difference in the type or number of nutritional supplements consumed according to food allergy status (Table 1).

\section{Nutrient intakes of adolescents}

Table 2 shows nutrient intakes of adolescents, including dietary supplements, stratified by food allergy status. Food allergic adolescents consumed a significantly lower \% energy from fat, but higher \% energy from carbohydrate than control participants $(\mathrm{p}<0.05)$, although both groups were very close to national recommendations ${ }^{(34)}$ of $50 \%$ and $35 \%$ for carbohydrate and fat respectively. Intakes of niacin and selenium were higher in food allergic than control participants. There was no difference in intake of any other nutrient according to food allergy status. Vitamin D intake was higher in food allergic than control participants (6.2 $\mu \mathrm{g}$ vs 3.3 $\mu \mathrm{g}$ ), however the difference was not significant. Across both food allergic and control adolescents, intakes of thiamine, vitamin B12 and vitamin C were in excess of $250 \%$ the RNI.

Figure 2 shows the percentage of adolescents consuming less than the RNI for micronutrients (including dietary supplements). All participants met the RNI for phosphorous. Intakes of potassium, magnesium and selenium were particularly low, with $81 \%, 74 \%$ and $80 \%$ of adolescents consuming less than the RNI respectively. Likewise, a substantial proportion of all adolescents had suboptimal intakes of calcium, iron, zinc, and iodine; with 48\%, 60\%, $51 \%$ and $55 \%$ not meeting the RNI respectively. There was no difference in the proportions of participants not meeting the RNI according to food allergy status for any nutrient. Vitamin D is not shown in the Figure as at the time there was no RNI for vitamin D for this age group. Using the current vitamin $\mathrm{D}$ recommendation of $10 \mu \mathrm{g}$ per day for those $>1$ year old ${ }^{(35)}$, only 
$4(8.3 \%)$ adolescents with food allergy met the RNI, with 1 (3\%) adolescent control participants meeting the RNI; all of whom took dietary supplements.

Nutrient intake of adolescents excluding dietary supplements

Supplementary Table 1 shows the nutrient intake of adolescents, excluding dietary supplements. Overall removing dietary supplements from the analysis did not alter any of the main findings. Intakes of niacin and selenium were higher in food allergic than control participants. There was no difference in intake of any other nutrient according to food allergy status. Intakes of thiamine, vitamin B12 and vitamin C were in excess of $250 \%$ the RNI. There was no difference in the proportions of participants not meeting the RNI according to food allergy status for any nutrient.

\section{Nutrient intake of adults}

Overall, looking across all adult participants, potassium and selenium intakes did not meet the recommended levels. Adults with food allergies had significantly higher intakes of folate and zinc than those without, as shown in Table 2.

Examining the percentages of adults below the RNI (Figure 3), there was a substantial number of adults who failed to consume the RNI for potassium (70\%) and selenium (84.2\%). Other nutrients that were consumed in suboptimal amounts by around half of all adults included magnesium (40\% below RNI), iron (55.7\% below RNI) and iodine (48.6\% below RNI). There were significantly more control participants (91.5\%) than food allergic participants $(69.6 \%)$ with intakes of selenium below the RNI $(\mathrm{p}<0.05)$. Using the current vitamin D recommendation of $10 \mu \mathrm{g}$ per day for those $>1$ year old ${ }^{(35)}$, only 1 (4.5\%) adult with food allergy and only $2(4.2 \%)$ control participants met the RNI, all of whom took dietary supplements.

Nutrient intake of adults excluding dietary supplements

Supplementary Table 1 shows the nutrient intake of adults, excluding dietary supplements. There was no difference in nutrient intake between those with and without food allergy (i.e. the higher intake of folate and zinc observed in adults with food allergy could be attributed to intake from dietary supplements). 


\section{Discussion}

To our knowledge this is the first study to investigate the full spectrum of nutrient intakes in food-allergic adolescents and adults, compared to a control group of healthy participants. Overall we have demonstrated higher intakes of certain micronutrients in food allergic participants (niacin and selenium in adolescents; folate and zinc in adults). The differences in adults can be attributed to dietary supplements. Although the dietary intake of food allergic participants was no worse than controls, suboptimal intakes of several micronutrients, especially minerals, were observed across all participants. Of note, we did not observe any difference in calcium intake between groups, which has been highlighted as a key nutrient in paediatric studies ${ }^{(5,6,11)}$. Paediatric studies have attributed particular nutrients to specific excluded foods (e.g. calcium ${ }^{(7)}$ or iodine ${ }^{(10)}$ to cows' milk) however due to the variety of food exclusion diets included in this study and the wide distribution of some nutrients in a range of foods (e.g. selenium, niacin), it is not possible to attribute each micronutrient to the exclusion of a specific food. Unlike most paediatric studies ${ }^{(5,6,8,12,13,36)}$, we did not observe any difference in BMI according to food allergy status.

In adolescents, differences in nutrient intake between the food-allergic and the control group were confined to fat, carbohydrate, niacin, and selenium. Although energy intake between adolescents with and without food allergies was similar, the percentage of energy derived from fat was lower and from carbohydrate intake higher among the food allergic group. One possible reason for this might be that adolescents with food allergies are less likely to consume high fat confectionary and snack foods, as many of these products have traces of food allergens (e.g. peanut). Previous research has suggested that adolescents and families with food allergies find it difficult to source prepacked foods without allergen traces labelling ${ }^{(37)}$. However the differences in macronutrient percentage derived from energy should not be overstated. Both groups' intakes were similar to recommended levels, therefore the differences are unlikely to be clinically meaningful. Looking at micronutrients that differed by food allergic status; niacin is widely distributed in foods ${ }^{(24)}$, therefore a higher intake cannot necessarily be attributed to a particular food group. A higher intake of selenium has previously been reported in infants consuming a milk-free diet ${ }^{(38)}$, which may be explained by the selenium content of substitute soya products ${ }^{(24)}$. Only one previous study of adolescents was identified in the literature, which did not show any difference in nutritional intake in 12-19 year olds ${ }^{(15)}$. 
In adult participants, differences existed solely in zinc and folate intake, with the food allergic group having higher intakes of both. However differences were only apparent when dietary supplements were factored in. Due to the paucity of literature related to nutrient intake in adults with food allergy, it is not possible to compare our data directly to other studies. Kim et al. ${ }^{(15)}$ demonstrated higher intakes of calcium, iron, folate and vitamin $\mathrm{E}$ in adults with food allergy, however they included participants with allergies to atypical foods such as beef, pork, and chicken; the control participants had atopic dermatitis and the contribution of dietary supplements was not clear. Nachshon et al. ${ }^{(16)}$ highlighted that young adults with milk allergy were at greater risk for osteoporosis due to lower intakes of calcium and phosphorous, however we did not detect a difference in these nutrients, which may be because we recruited adults with allergies to a range of foods, not solely to milk.

This study assessed nutrient intake from both food sources and dietary supplements. The percentage of supplement use in this study was 21.2 to $34.8 \%$, depending on age and food allergic status. Data indicate that some participants with, but also without food allergies, would not have met the recommendations without dietary supplements, particularly for several minerals. For calcium, it has been shown among children with food allergies ${ }^{(5,6,11)}$ and adolescents on a cows' milk free diet ${ }^{(39)}$ that these recommendations cannot be met without use of fortified foods, infant formula or dietary supplements. However, Meyer et al. ${ }^{(40)}$ have shown that even with supplementation deficiency can occur, as can excess intakes; thus underlying the importance of individualised dietetic advice. It is difficult to make generalised recommendations on nutritional supplementation of adolescents and adults with food allergy, due to the wide spectrum of foods implicated and differing severity of symptoms. This study is novel and therefore needs to be replicated in other settings in order to establish if patterns of nutrition deficiencies exist.

Overall, a large number of participants with and without food allergies did not meet the RNI for a range of minerals. One explanation could be the underestimation of energy intake. If energy intake is underestimated, it is possible that the intake of other nutrients is also underestimated ${ }^{(41)}$. The percentage of under-reporters was higher among adolescents than adults (see Table 1). However, the observed low nutrient intakes might not only be a result of under-reporting, but rather a matter of poor food choice and low intake of nutrientdense foods. 
We recognise some limitations in this study. There were differences in the mean age of the food allergic and control adult group, however both groups mean age were in their 30s. There was a greater proportion of males in the food allergy adolescent group, however this would not have had an effect on the nutrient intake analysis, which was compared to gender specific Dietary Recommended Values (DRVs). The food-allergic participants recruited were predominantly members of a support charity, which arguably could have attracted individuals with high socio-economic status and more severe food allergies who have received specialised dietetic advice. The majority of control participants were recruited through advertisements on university websites, therefore possibly attracting health motivated participants. Furthermore, the study sample was recruited from Portsmouth and the Isle of Wight and may not be representative of the general population living in the UK. Due to the wide spectrum of food allergies it was not possible to link specific foods with specific nutrient deficiencies within our sample. Although the food diary was considered as the most appropriate dietary assessment method for this study, its validity should have ideally been assessed prior to its use. However, validation studies are relatively expensive and difficult to perform. All food diaries were carefully checked and participants were prompted for missing details over the phone. Overall this study was suitably powered, with 151 participants returning completed food diaries, which compares very favourably to other similar studies.

\section{Conclusion}

This study provides a comprehensive survey of nutrient intake in adolescents and adults with food allergies. It shows that dietary intake \& BMI between the food-allergic and the general population is largely similar and in some cases dietary intake was better in individuals with food allergy. However, a substantial proportion of all participants did not meet RNIs for micronutrients, particularly minerals, which may indicate poor choice of foods with low nutrient density. This underlines the importance of individualised dietetic involvement in the management of food allergies.

\section{TRANSPARENCY DECLARATION}

The lead author affirms that this manuscript is an honest, accurate, and transparent account of the study being reported, that no important aspects of the study have been omitted and that any discrepancies from the study as planned (and registered with) have been explained. The reporting of this work is compliant with STROBE guidelines. 


\section{Acknowledgements}

We would like to thank the Anaphylaxis Campaign, local allergy clinics and schools for their help in recruitment, and Maja Berry and Leanne Smith for assistance in data entry. Funding for this study was received from the University of Portsmouth, UK, as part of a studentship grant. The authors declare that they have no conflicts of interest. 


\section{References}

1. Johansson SGO, Bieber T, Dahl R, et al. (2004) Revised nomenclature for allergy for global use: Report of the Nomenclature Review Committee of the World Allergy Organization, October 2003. J. Allergy Clin. Immunol. 113, 832-836.

2. Nwaru BI, Hickstein L, Panesar SS, et al. (2014) Prevalence of common food allergies in Europe: A systematic review and meta-analysis. Allergy Eur. J. Allergy Clin. Immunol. 69, 992-1007.

3. Boyce JA, Assa'ad A, Burks AW, et al. (2010) Guidelines for the diagnosis and management of food allergy in the United States: report of the NIAID-sponsored expert panel. In J. Allergy Clin. Immunol., vol. 126, pp. S1-S58.

4. Venter, Laitinen K \& Vlieg-Boerstra B (2012) Nutritional Aspects in Diagnosis and Management of Food Hypersensitivity—The Dietitians Role. J. Allergy 2012, 1-11.

5. Flammarion S, Santos C, Guimber D, et al. (2011) Diet and nutritional status of children with food allergies. Pediatr. Allergy Immunol. 22, 161-165.

6. Christie L, Hine RJ, Parker JG, et al. (2002) Food allergies in children affect nutrient intake and growth. J. Am. Diet. Assoc. 102, 1648-1651.

7. Henriksen C, Eggesbø M, Halvorsen R, et al. (2000) Nutrient intake among two-yearold children on cows' milk-restricted diets. Acta Paediatr. 89, 272-278.

8. Robbins KA, Wood RA \& Keet CA (2014) Milk allergy is associated with decreased growth in US children. J. Allergy Clin. Immunol. 134, 1466-1468.e6. American Academy of Allergy, Asthma \& Immunology.

9. Vieira MC, Morais MB, Spolidoro JVN, et al. (2010) A survey on clinical presentation and nutritional status of infants with suspected cow' milk allergy. BMC Pediatr. 10, 25.

10. Thomassen R, Kvammen J, Eskerud M, et al. (2017) Iodine Status and Growth in 0-2Year-Old Infants With Cow's Milk Protein Allergy. J. Pediatr. Gastroenterol. Nutr. Epub ahead.

11. Berry MJ, Adams J, Voutilainen H, et al. (2015) Impact of elimination diets on growth and nutritional status in children with multiple food allergies. Pediatr. Allergy Immunol. 26, n/a-n/a.

12. Meyer R, De Koker C, Dziubak R, et al. (2014) Malnutrition in children with food allergies in the UK. J. Hum. Nutr. Diet. 27, 227-235.

13. Mehta H, Ramesh M, Feuille E, et al. (2014) Growth Comparison in children with and without food allergies in 2 different demographic populations. J. Pediatr. 165, 842848. 
14. Sova C, Feuling MB, Baumler M, et al. (2013) Systematic Review of Nutrient Intake and Growth in Children with Multiple IgE-Mediated Food Allergies. Nutr Clin Pr. 28, $669-675$.

15. Kim J, Kwon J, Noh G, et al. (2013) The effects of elimination diet on nutritional status in subjects with atopic dermatitis. 7, 488-494.

16. Nachshon L, Goldberg MR, Schwartz N, et al. (2013) Decreased bone mineral density in young adult IgE-mediated cow's milk - allergic patients. J. Allergy Clin. Immunol. 134, 1108-1113.e3. Elsevier Ltd.

17. Skypala IJ, Bull S, Deegan K, et al. (2013) The prevalence of PFS and prevalence and characteristics of reported food allergy; a survey of UK adults aged 18-75 incorporating a validated PFS diagnostic questionnaire. Clin. Exp. Allergy 43, 928940.

18. Das JK, Salam RA, Thornburg KL, et al. (2017) Nutrition in adolescents: physiology, metabolism, and nutritional needs. Ann. N. Y. Acad. Sci. 1393, 21-33.

19. Patton GC, Sawyer SM, Santelli JS, et al. (2016) Our future: a Lancet commission on adolescent health and wellbeing. Lancet (London, England) 387, 2423-2478.

20. Chaudhry RQ \& Oppenheimer JJ (2012) Update on Food Allergy in Adults. 311-320.

21. Sommer I, MacKenzie H, Venter C, et al. (2012) Factors influencing food choices of food-allergic consumers: Findings from focus groups. Allergy Eur. J. Allergy Clin. Immunol. 67, 1319-1322.

22. Sommer I, MacKenzie H, Venter C, et al. (2014) An exploratory investigation of food choice behavior of teenagers with and without food allergies. Ann. Allergy Asthma Immunol. 112, 446-452.

23. Nelson M, Atkinson M \& Meyer J (1997) Food portion sizes: A Photographic Atlas. London: MAFF Publications.

24. Food Standards Agency (2002) McCance and Widdowson's The Composition of Foods. 6th editio [Church S, editor]. Cambridge: Royal Society of Chemistry.

25. Food Standards Agency (2002) Food portion sizes. 3rd editio. London: The Stationary Office.

26. Wrieden WL, Longbottom PJ, Adamson AJ, et al. (2008) Estimation of typical food portion sizes for children of different ages in Great Britain. Br. J. Nutr. 99, 1344-1353.

27. Von Mutius E (1996) Epidemiology of asthma: ISAAC--International Study of Asthma and Allergies in Childhood. Pediatr Allergy Immunol 7, 54-6.

28. Goldberg G, Black A, Jebb S, et al. (1991) Critical evaluation of energy intake data 
using fundamental principles of energy physiology: 1. Derivation of cut-off limits to identify under-recording. Eur. J. Clin. Nutr. 45, 569-581.

29. Black A (2000) Critical evaluation of energy intake using the Goldberg cut-off for energy intake:basal metabolic rate. A practical guide to its calculation, use and limitations. Int. J. Obes. 24, 1119-1130.

30. Schofield WN (1985) Predicting basal metabolic rate, new standards and review of previous work. Hum. Nutr. Clin. Nutr. 39 Suppl 1, 5-41.

31. Black A (2000) The sensitivity and speci®city of the Goldberg cut-off for EI:BMR for identifying diet reports of poor validity. Eur. J. Clin. Nutr. 54, 395-404.

32. McGowan M \& Gibney MJ (1993) Calcium intakes in individuals on diets for the management of cows' milk allergy: a case control study. Eur. J. Clin. Nutr. 47, 609616.

33. Mehta H, Groetch M \& Wang J (2013) Growth and nutritional concerns in children with food allergy. Curr. Opin. Allergy Clin. Immunol. 13, 275-9.

34. Committee on Medical Aspects of Food and Nutrition Policy (COMA) (1991) Dietary reference values for food energy and nutrients for the United Kingdom. Report of the panel on Dietary Referencce Values. Report on Health and Social Subjects number 41. London: .

35. Scientific Advisory Committee on Nutrition (2016) Vitamin D and health. .

36. Berni Canani R, Leone L, D’Auria E, et al. (2014) The Effects of Dietary Counseling on Children with Food Allergy: A Prospective, Multicenter Intervention Study. J. Acad. Nutr. Diet. 114, 1432-1439. Elsevier.

37. Polloni L, Toniolo A, Lazzarotto F, et al. (2013) Nutritional behavior and attitudes in food allergic children and their mothers. Clin. Transl. Allergy 3, 41.

38. Maslin K, Oliver EM, Scally KS, et al. (2016) Nutritional adequacy of a cows' milk exclusion diet in infancy. Clin. Transl. Allergy 6, 20. BioMed Central.

39. Gao X, Wilde PE, Lichtenstein AH, et al. (2006) Meeting Adequate Intake for Dietary Calcium without Dairy Foods in Adolescents Aged 9 to 18 Years (National Health and Nutrition Examination Survey 2001-2002). J. Am. Diet. Assoc. 106, 1759-1765.

40. Meyer R, De Koker C, Dziubak R, et al. (2015) A practical approach to vitamin and mineral supplementation in food allergic children. Clin. Transl. Allergy 5, 5-11.

41. Livingstone MBE \& Black AE (2003) Markers of the validity of reported energy intake. J. Nutr. 133, 895S-920S. 
Table 1: Characteristics of adolescents and adults with food allergies and controls $(n=151)$

\begin{tabular}{|c|c|c|c|c|}
\hline & $\begin{array}{c}\text { Adolescents } \\
\text { with FA } \\
(\mathrm{n}=50)\end{array}$ & $\begin{array}{c}\text { Adolescents } \\
\text { without FA } \\
\quad(n=33)\end{array}$ & $\begin{array}{c}\text { Adults with } \\
\text { FA } \\
(\mathrm{n}=23)\end{array}$ & $\begin{array}{c}\text { Adults } \\
\text { without FA } \\
(\mathrm{n}=47)\end{array}$ \\
\hline Age (y); (mean, SD) & $14.5(2.4)$ & $14.5(2.2)$ & $39.4(13.7)^{*}$ & $30.2(9.5)$ \\
\hline Male (n, \%) & $33(66.0)^{*}$ & $12(36.4)$ & $4(17.4)$ & $9(19.1)$ \\
\hline White ethnicity (n, \%) & $43(87.8)$ & $29(87.9)$ & $22(95.7)$ & $38(80.9)$ \\
\hline BMI $\left(\mathrm{kg} / \mathrm{m}^{2}\right) ;($ mean, SD) & $20.1(3.6)$ & $20.3(3.4)$ & $24.5(4)$ & $24.2(4.5)$ \\
\hline Graduate/postgraduate education^ ${ }^{\wedge}$ & $25(50.0)$ & $22(68.8)$ & $12(52.2)$ & $28(59.6)$ \\
\hline Urban living location (n, \%) & $35(70.0)$ & $25(78.1)$ & $18(78.3)$ & $41(87.2)$ \\
\hline Under-reporting (Goldberg) (n, \%) & $14(29.2)$ & $9(27.3)$ & $5(21.7)$ & $8(17.0)$ \\
\hline Irregular recording $^{\mathrm{b}}(\mathrm{n}, \%)$ & $15(30.0)$ & $5(15.2)$ & $3(13.0)$ & $8(17.4)$ \\
\hline Supplement use (n, \%) & $12(24.0)$ & $7(21.2)$ & $8(34.8)$ & $11(23.4)$ \\
\hline $\begin{array}{l}\text { Multivitamin and minerals } \\
(\mathrm{n}, \%)\end{array}$ & $5(10.0)$ & $5(15.2)$ & $6(26.1)$ & $6(12.8)$ \\
\hline $\begin{array}{l}\text { Single/Few vitamins or } \\
\text { minerals }(\mathrm{n}, \%)\end{array}$ & $3(6.0)$ & $1(3.0)$ & $3(13.0)$ & $6(12.8)$ \\
\hline Probiotics $(\mathrm{n}, \%)$ & $1(2.0)$ & 0 & $3(13.0)$ & 0 \\
\hline Fish oil $(\mathrm{n}, \%)$ & $5(10.2)$ & $1(3.0)$ & $2(8.7)$ & $3(6.4)$ \\
\hline
\end{tabular}

FA: Food allergy. *significant difference between food allergy and control group $(\mathrm{p}<0.05)$

$\wedge$ for adolescents, maternal education level was used. 
Table 2: Daily nutrient intakes of adolescent and adults with food allergies and their controls, including dietary supplements $(\mathrm{n}=151)$

\begin{tabular}{|c|c|c|c|c|}
\hline \multirow[t]{2}{*}{ Nutrients } & \multicolumn{2}{|c|}{ Adolescents } & \multicolumn{2}{|c|}{ Adults } \\
\hline & $\begin{array}{l}\text { Food allergic } \\
\quad(n=48)\end{array}$ & $\begin{array}{l}\text { Control } \\
(\mathrm{n}=33)\end{array}$ & $\begin{array}{l}\text { Food allergic } \\
(n=23)\end{array}$ & $\begin{array}{l}\text { Control } \\
(\mathrm{n}=47)\end{array}$ \\
\hline & \multicolumn{2}{|c|}{ Mean (SD) } & \multicolumn{2}{|c|}{ Mean (SD) } \\
\hline Energy (\%EAR) & $88.2(20.9)$ & $89.3(15.7)$ & $95.4(21.4)$ & $99.4(21.7)$ \\
\hline Protein (\%RNI) & $166.9(46.9)$ & $164.4(37.8)$ & $169.9(47.4)$ & $169.7(57.2)$ \\
\hline Fat $(\mathrm{g})$ & $73.9(24.9)$ & $77.1(18.4)$ & $70.1(20.1)$ & $80.1(24.8)$ \\
\hline$\%$ Total energy & $33.1(5.5)^{*}$ & $36.7(5.2)$ & $32.9(5.9)$ & $35.1(5.5)$ \\
\hline Carbohydrates (g) & $258.3(62.6)$ & $236.8(47.1)$ & $241.2(101.2)$ & $250.6(56.9)$ \\
\hline$\%$ Total energy & $50.8(6.4)^{*}$ & $47.1(4.9)$ & $46.4(11.0)$ & $46.6(5.3)$ \\
\hline NSP (g) & $13.7(5.0)$ & $13.1(3.7)$ & $16.4(6.3)$ & $15.4(5.6)$ \\
\hline Vitamin A (\%RNI) & $145.1(83.9)$ & $129.3(61.8)$ & $195.5(155.9)$ & $191.3(182.2)$ \\
\hline Vitamin C (\%RNI) & $387.7(260.6)$ & $326.1(240.8)$ & $415.4(608.8)$ & $333.0(409.2)$ \\
\hline Vitamin $\mathrm{D}(\mu \mathrm{g})^{\wedge}$ & $6.2(18.1)$ & $3.3(2.6)$ & $3.4(2.8)$ & $3.0(2.7)$ \\
\hline Vitamin $\mathrm{E}(\mathrm{mg})^{\wedge}$ & $9.5(9.0)$ & $8.9(5.6)$ & $20.1(37.1)$ & $12.0(15.6)$ \\
\hline Thiamine (\%RNI) & $273.4(379.3)$ & $380.8(625.6)$ & $375.9(410.4)$ & $271.7(257.0)$ \\
\hline Riboflavin (\%RNI) & $195.3(250.0)$ & 163.7 (96.2) & $247.4(251.3)$ & $174.6(97.9)$ \\
\hline Niacin equiv. (\%RNI) & $248.4(73.5)^{*}$ & $189.2(59.3)$ & $245.7(89.2)$ & $233.8(63.9)$ \\
\hline Vitamin B6 (\%RNI) & $190.8(119.0)$ & $179.6(71.0)$ & $251.7(210.7)$ & $190.5(165.8)$ \\
\hline Vitamin B12 (\%RNI) & $341.7(253.7)$ & $324.0(211.1)$ & $385.4(297.2)$ & $352.9(277.4)$ \\
\hline Folate (\%RNI) & $128.6(53.2)$ & $134.9(56.9)$ & $170.5(73.5)^{*}$ & $136.3(55.9)$ \\
\hline Sodium (\%RNI) & $163.4(58.6)$ & $160.0(34.2)$ & $178.6(56.7)$ & $211.3(101.9)$ \\
\hline Potassium (\%RNI) & $84.5(24.5)$ & $80.1(23.2)$ & $94.2(40.8)$ & $88.6(25.1)$ \\
\hline Calcium (\%RNI) & $104.8(49.1)$ & $104.7(29.4)$ & $132.4(43.3)$ & $135.0(50.7)$ \\
\hline Magnesium (\%RNI) & $89.9(29.4)$ & $88.3(26.2)$ & $113.7(37.9)$ & $108.9(29.7)$ \\
\hline Phosphorus (\%RNI) & $178.3(57.8)$ & $184.4(38.3)$ & $247.7(64.1)$ & $247.3(62.6)$ \\
\hline Iron (\%RNI) & $104.3(50.2)$ & $87.9(40.3)$ & $125.1(59.7)$ & $103.0(50.1)$ \\
\hline Copper (\%RNI) & $128.9(50.6)$ & $137.7(58.4)$ & $117.4(46.2)$ & $110.4(33.9)$ \\
\hline Zinc (\%RNI) & $110.6(54.3)$ & $106.3(29.8)$ & $157.6(53.6)^{*}$ & $134.9(40.6)$ \\
\hline Selenium (\%RNI) & $86.6(49.7)^{*}$ & $64.4(35.2)$ & $75.4(60.0)$ & $61.8(33.6)$ \\
\hline Iodine (\%RNI) & $111.1(84.1)$ & $112.3(56.3)$ & $107.1(52.5)$ & $114.7(52.2)$ \\
\hline
\end{tabular}

EAR: Estimated Average Requirement. RNI: Reference Nutrient Intake. NSP: Non Starch Polysaccharide *significant difference between food allergic and control groups $(p<0.05)$

${ }^{\wedge}$ No dietary reference values existed for vitamins $\mathrm{D}$ and $\mathrm{E}$ at the time of the study in the age groups studied 


\section{Figure legends}

Figure 1: Recruitment of participants

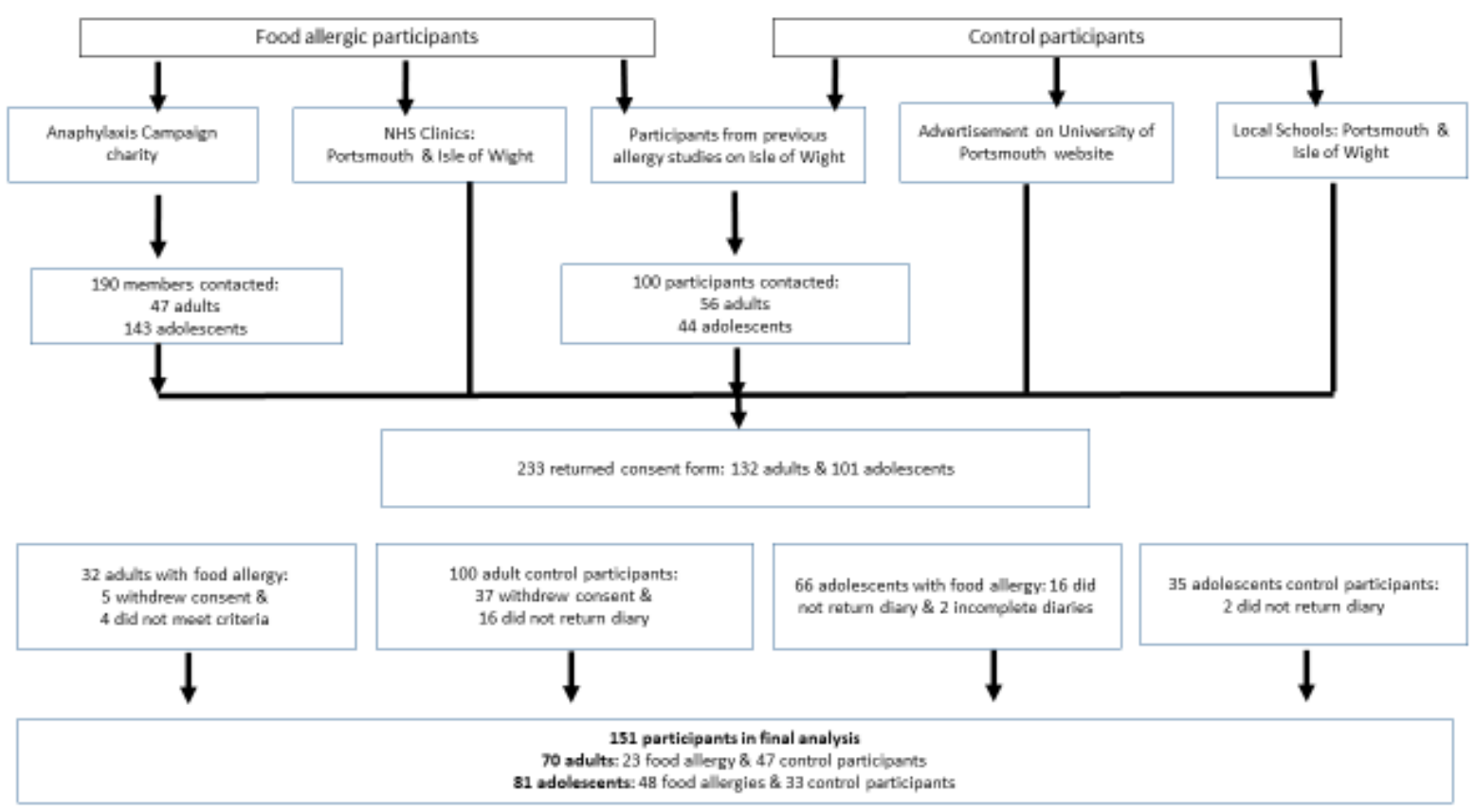


Figure 2: Percentage of adolescent participants not achieving RNI for micronutrients

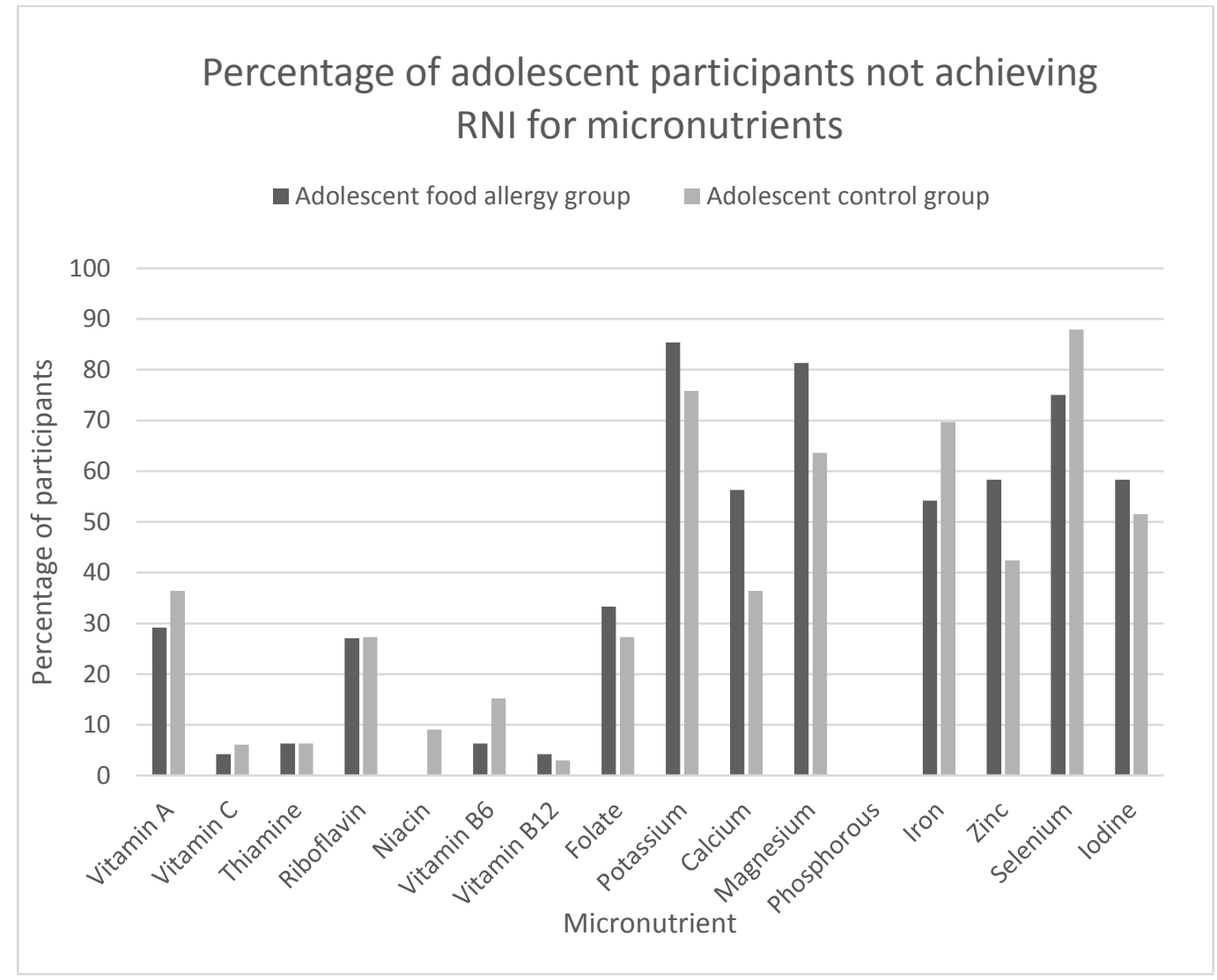


Figure 3: Percentage of adult participants not achieving RNI for micronutrients

Percentage of adult participants not achieving RNI for micronutrients

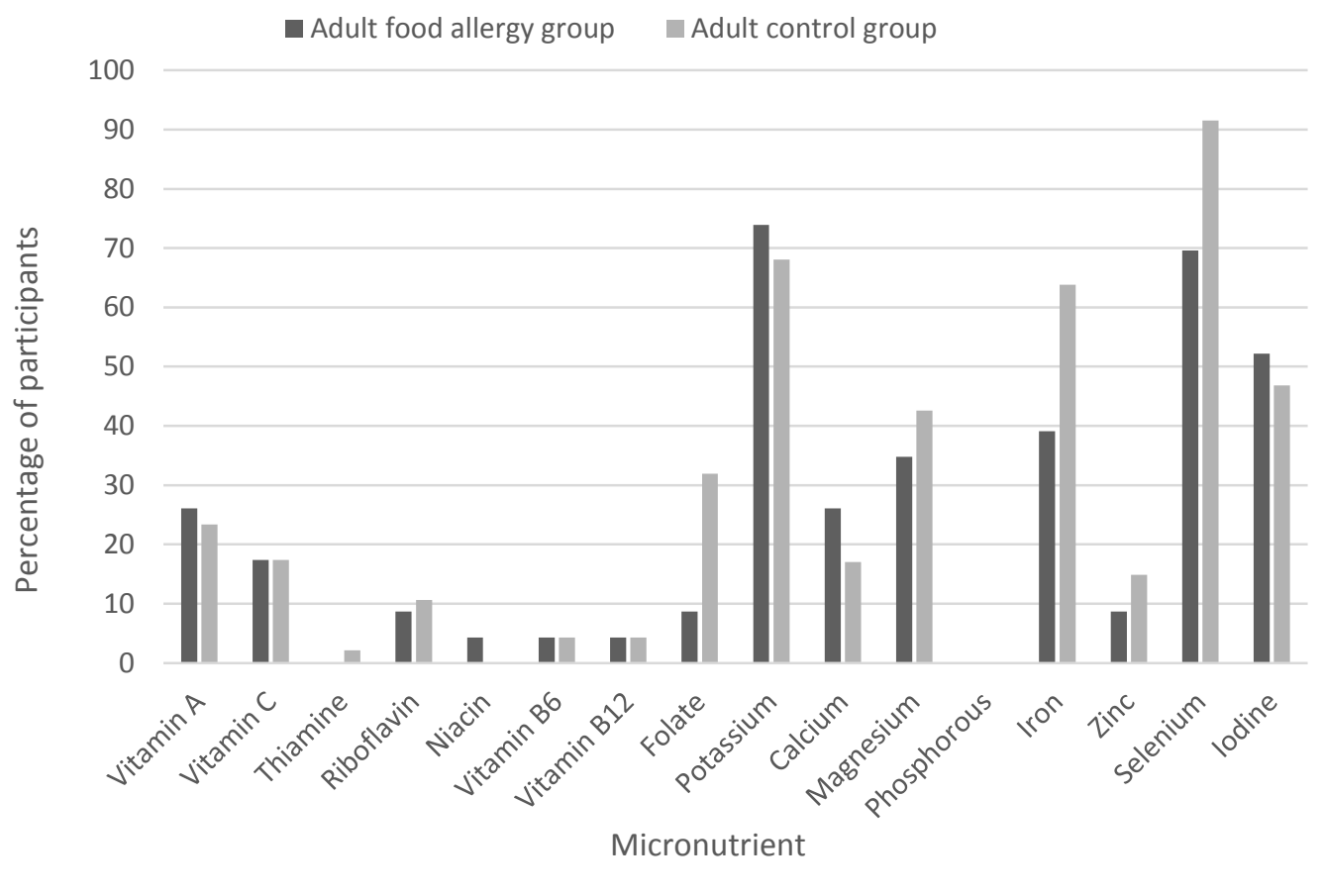


